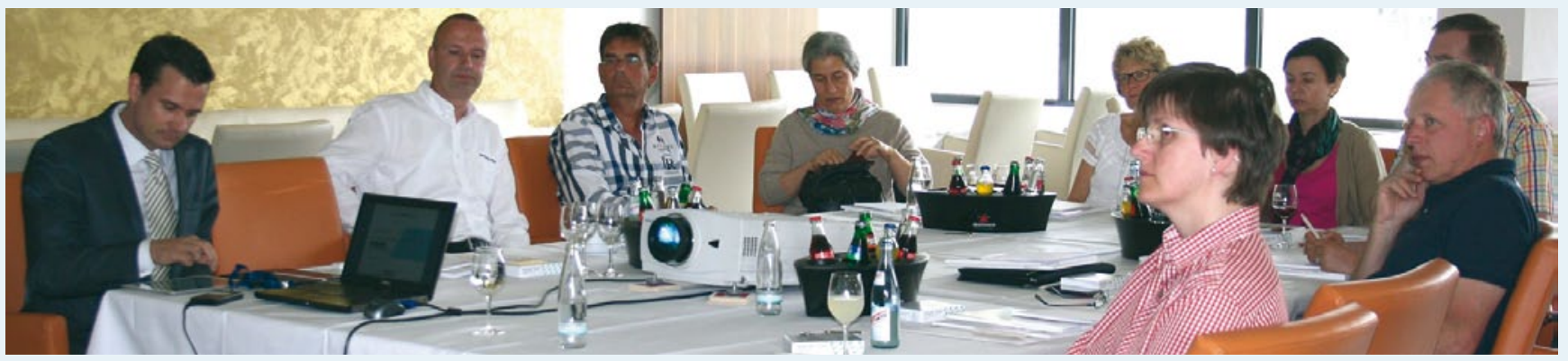

Basisversorgung

\title{
Seminar vermittelt Möglichkeiten für eine neue Strategie
}

Im Rahmen eines Seminars mit demTitel „EQUIA - Wir rütteln an der Basis“ machten sich interessierte Zahnärzte am 30. Mai 2012 in Paderborn ein eigenes Bild von den Möglichkeiten des 2-stufigen Füllungskonzepts EQUIA (GC Germany, Bad Homburg) in der Basisversorgung. Das Zahntechniklabor Kluthe hatte hierfür gemeinsam mit GC erfahrene Referenten aus der Praxis gewinnen können, die aus ihrer jeweiligen Warte die Vorteile des 2-stufigen Konzepts darstellten. Im 1. Teil des Seminars kam Zahnarzt Gert Fecht zu Wort, welcher seit über 10 Jahren selbst eine Praxis in Uplengen betreibt und sich in seinem Vortrag stark am Behandler orientierte. Nach Gebrauchsanweisung des Herstellers indiziert für Kavitäten der Klasse I und kleinere Kavitäten der Klasse II gewährleistet das Material eine zahnfarbene und amalgamfreie Basisversorgung im kaudruckbelasteten Seitenzahnbereich. Fecht führte weiterhin die grundlegenden Vorteile des 2-stufigen Füllungskonzepts an, die in der einfachen und schnellen Verarbeitung, den physikalischen Eigenschaften sowie der Abrechenbarkeit durch die Gesetzlichen Krankenversicherungen (GKV) liegen. Als Fachanwalt für Medizinund Arztrecht berät Jens Pätzold bundes- weit Zahnärzte - speziell im niedergelassenen Bereich - und stand dem Seminar mit entsprechender Expertise zur Verfügung. Im Rahmen des Seminars wurden jedoch nicht nur die Inhalte geradlinig vermittelt, sondern auch sehr rege und aufschlussreiche Diskussionen unter den Beteiligten geführt - wobei unter anderem viele Fragen und Anregungen zur praktischen Anwendung und Verarbeitung von EQUIA gestellt wurden.

Nach einer Pressemitteilung der

dNA - dentale Nachrichten-Agentur GmbH,

Bad Homburg

E-Mail: info@d-n-a.eu

\section{Download}

\section{Abrechnungsempfehlungen Endodontie}

Die strikte Anwendung der Kons-Richtlinie 9 hat dazu geführt, dass Wurzelkanalbehandlungen aufgrund der einschränkenden Kriterien seltener als Kassenleistung erbracht werden können. Werden die BEMA-Richtlinien erfüllt, gibt es einige Möglichkeiten zur Vereinbarung von Zusatzleistungen nach GOZ, die nicht unter das Zuzahlungsverbot fallen. Dazu gehören z.B. die elektronische Längenbe-
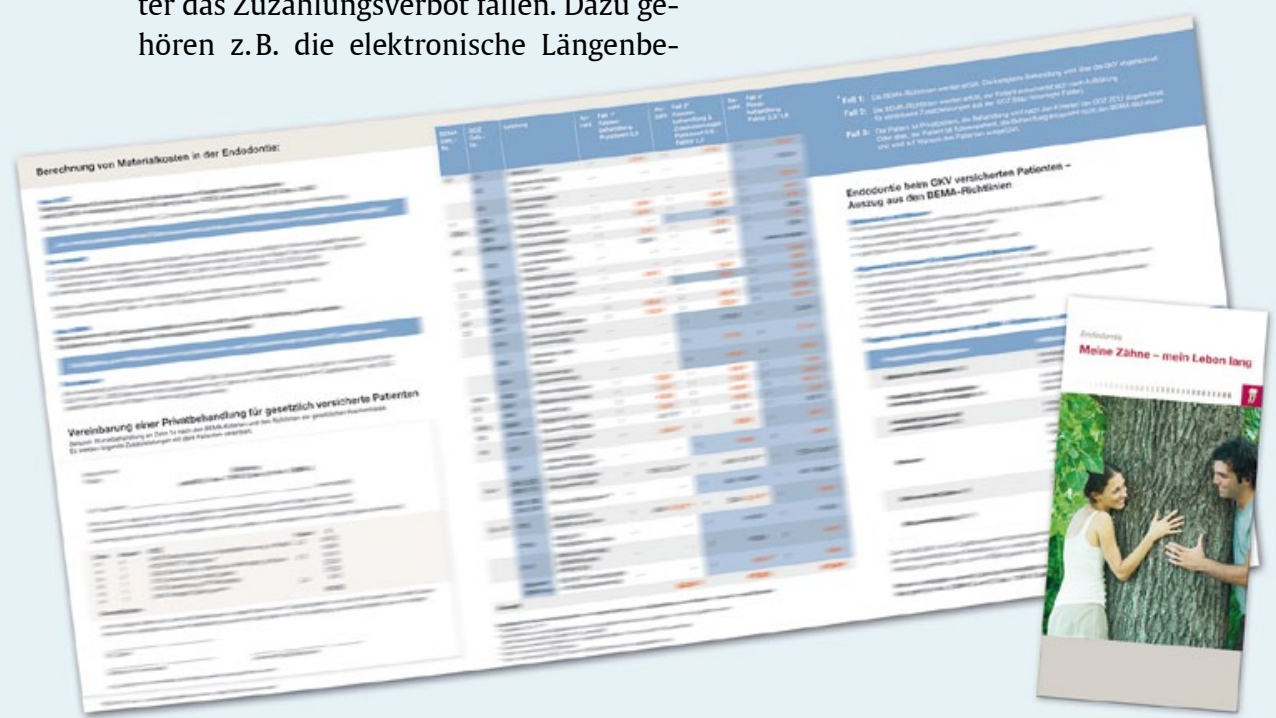

stimmung oder der adhäsive Stiftaufbau. Ist die endodontische Behandlung sinnvoll, also mit guter Prognose für den Erhalt des Zahnes, entspricht aber nicht den strengen BEMA-Richtlinien, kann auch beim GKV-Patienten nach GOZ 2012 abgerechnet werden. Die gesonderte Berechnung von Materialkosten für nur ein- mal verwendbare Nickel-Titan-Instrumente ist nach GOZ ebenfalls möglich. In Zusammenarbeit mit ZAB Zahnmedizinischer Abrechnungs- und Beratungsservice hat VDW München die BEMA-Kriterien und Abrechnungsmöglichkeiten nach BEMA und GOZ 2012 mit einem Mustertext für die Vereinbarung einer Privatbehandlung beim GKV-Patienten sorgfältig zusammengestellt und präsentiert diese kompakt und übersichtlich auf wenigen Seiten. In tabellarischer Form werden die Möglichkeiten der Abrechnung als reine Kassenbehandlung, Kasse plus Zusatzleistung und Privatliquidation nebeneinander dargestellt.

Ergänzend dazu gibt es die Patienteninformations-Broschüre „Meine Zähne mein Leben lang“. Beide Broschüren können als PDF von der Internetseite www. vdw-dental.com heruntergeladen oder kostenlos bestellt werden: E-Mail: info@ vdw-dental.com oder Tel.: 089/627340.

Nach einer Pressemitteilung der

VDW GmbH, München 


\section{Firmenübernahme \\ Sortiment erweitert}

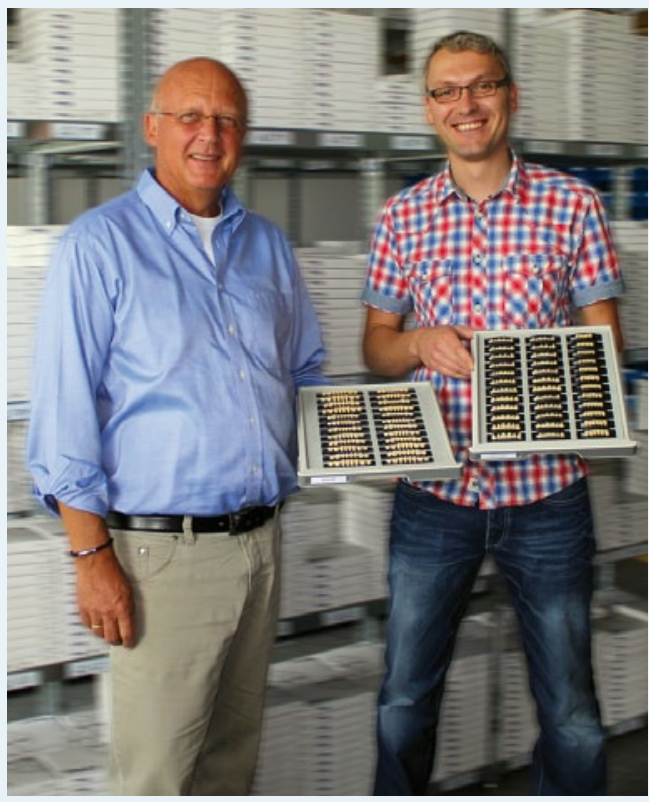

Die Düsseldorfer ARGEN Edelmetalle $\mathrm{GmbH}$ hat sich strategisch neu ausgerichtet und eine bedeutende Sortimentserweiterung mit dem Erwerb der INKA $\mathrm{GmbH}$ eingeleitet. Ab sofort bietet das Düsseldorfer Unternehmen auch Kunststoffzähne an und baut damit seine vorhandene Kompetenz weiter aus. ARGEN hat die INKA GmbH im Frühjahr dieses Jahres in das eigene Unternehmen integriert und den Firmensitz an den ARGEN-Standort nach Düsseldorf verlegt. Damit kommen die INKA-Front- und Seitenzähne aus Kunststoff in das ARGENSortiment und werden ab sofort bundesweit angeboten. Für das Unternehmen bedeutet die Übernahme auch einen Gewinn für seine Edelmetall-Sparte. Die Zähne werden in 16 Farbtönen entsprechend dem Vita Classic-Farbsystem ${ }^{\odot}$ hergestellt und sind leicht kombinierbar mit Metallkeramik. Sie besitzen ausgezeichnete physikalische Eigenschaften und eine exzellente Farbtreue. Im Bereich partieller und totaler Kunststoffprothesen sowie Immediat- und Interims-Prothesen folgen sie den Regeln balancierter Okklusionskonzepte. Angeboten werden klassische OK-Frontzahnformen, oval, dreieckig und quadratisch, mit ästhetisch und funktionell darauf abgestimmten UK-Front- und Seitenzähnen.

Nach einer Pressemitteilung der ARGEN Edelmetalle GmbH, Düsseldorf Internet: www.argen.de

\section{Praxistest \\ Außergewöhnliche Hydro- philie, gute Fließfähigkeit und einfaches Handling}

Kettenbach hat mit Identium ein neuartiges Material für die Abformtechnik entwickelt: Vinylsiloxanether (VSXE). In diesem Produkt vereinen sich die besten Eigenschaften von A-Silikon und Polyether. Das Ergebnis ist eine außergewöhnliche Hydrophilie, ausgezeichnete Fließfähigkeit, Geruchs- und Geschmacksneutralität sowie die leichte Entformung. Kurzum: Komfort und Präzision für die moderne Zahnarztpraxis. Diese Vorteile bestätigt auch der kürzlich durchgeführte Test des US-Magazins „Dental Product Shopper“. In dessen Auftrag testeten 10 Zahnärzte mit einem gebündelten DentalErfahrungsschatz von insgesamt 208 Jahren das Produkt Identium. Sie beurteilten das Material nach 10 verschiedenen Kriterien hinsichtlich seiner Eigenschaften sowie der Anwender- und Patientenfreundlichkeit. Bewertet wurden Kriterien wie Handhabung, Fließfähigkeit und Detailgenauigkeit - mit einer Gesamtbewertung von 4,2 auf der 5-Punkte-Skala.

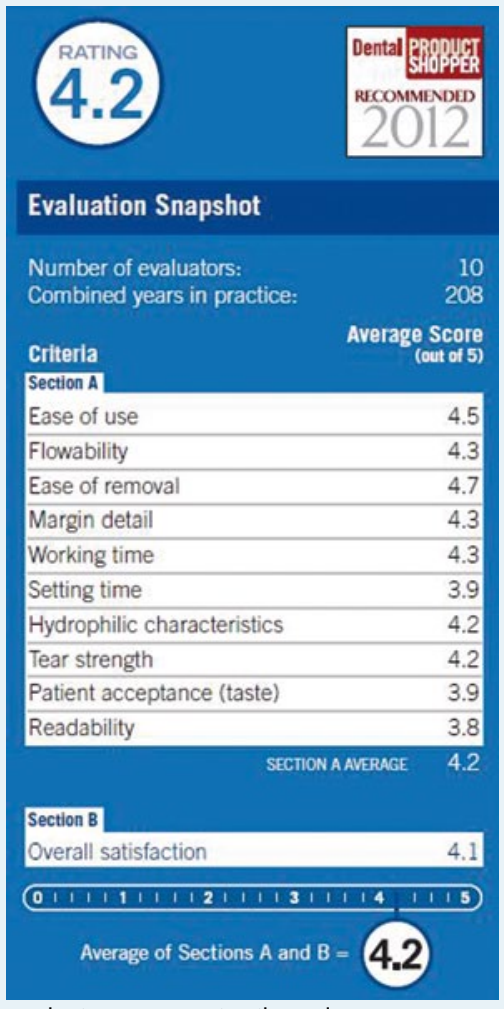

Nach einer Pressemitteilung der Kettenbach GmbH \& Co. KG, 35713 Eschenburg

Internet: www.kettenbach.de

\section{Abutments \\ Unterschiedliches Design für \\ Gingivamanagement}

Ab sofort bietet das Unternehmen Zfx Titanbasen für individuelle 2-teilige Abutments in 2 verschiedenen Ausführungen an. Die Regular-Design-Serie gleicht den Titanbasen anderer Hersteller und eignet sich aufgrund ihrer nach außen gewölbten Form insbesondere für Patienten mit dicker Gingiva (Biotyp B). Die zusätzliche Small-Design-Serie, die eine gerade Form aufweist und zur Gingiva hin verkürzt ist, führt zu vermindertem Druck auf das Weichgewebe und wird vor allem bei dünner Gingiva eingesetzt. Durch diese Auswahl sind die besten Voraussetzungen für ein optimales Weichgewebemanagement geschaffen.

Die Ausbildung des Gingivalsaums wird maßgeblich vom prothetischen Design beeinflusst, das sich somit auch auf die ästhetische Wirkung der gesamten Versorgung auswirkt. Durch ein nach außen gewölbtes Design - wie bei der Regular-Design-Serie der Titanbasen von Zfx - wird eine Konditionierung des Weichgewebes erzielt. Dies hat bei dicker Gingiva eine positive Wirkung auf die Ausformung des Gingivalsaums durch Verlagerung in apikale Richtung. Bei dünner Gingiva ist es hingegen wichtig, die Gingiva nicht zu konditionieren, um das bestehende Gewebevolumen und somit die gewünschte Lage des Gingivalsaums zu erhalten.

Dank der 2 unterschiedlichen Arten von Titanbasen, deren Kompatibilität mit allen gängigen Implantatsystemen garantiert werden kann, können Anwender des Zfx CAD/CAM-Systems nun je nach Anforderung und gewünschtem Resultat die entsprechende Lösung wählen.

Nach einer Pressemitteilung der

Zfx GmbH, Dachau

Internet: www.zfx-dental.com 


\section{Zeit und Kosten sparen}

Das ganzheitliche Hygienekonzept von Dürr Dental umfasst eine breite Palette von abgestimmten Systemlösungen aus einer Hand. Die Konzentrate und Gebrauchslösungen der System-Hygiene zur Desinfektion, Reinigung und Pflege decken alle Einsatzbereiche ab. Die einst in den Markt eingeführte durchgehende Farbcodierung vereinfacht die Anwendung im Praxisalltag: Blau für Instrumente, Grün für Flächen, Rosa für Haut und Hände sowie Gelb für Spezialberei- che (z.B. Sauganlagen, Mundspülbecken, Abdrücke etc.). Ergänzend ermöglichen innovative und speziell entwickelte Geräte und Technologien rationelles Arbeiten während der Instrumentenaufbereitung oder der Desinfektion von Flächen, Händen oder Abdrücken. Ein umfangreiches Service- und Schulungsangebot vervollständigt das ganzheitliche Hygienesystem von Dürr Dental. Neben der praxisgerechten Keimeliminierung setzt das Unternehmen alles daran, mikrobielle

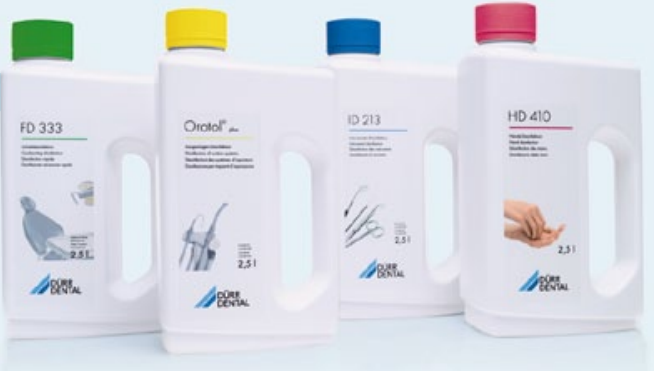

Kontaminationsketten bereits gar nicht erst entstehen zu lassen. Der Einsatz von berührungsfreier Sensortechnik bei der Entnahme und Dosierung von Präparaten ist ein wichtiges Beispiel dafür.

Nach einer Pressemitteilung der DÜRR DENTAL AG, Bietigheim-Bissingen Internet: www.duerr.de

\section{Lasertechnologie}

\section{Bindungen, die halten}

Lasertechnologie zum großen Nutzen für die Prothetik oder auch für die Kieferorthopädie wird nun seit 19 Jahren von der Firma Dentaurum angeboten. Das seinerzeit stark wachsende Interesse für Titan bedurfte einer besonderen Fügetechnik, die nur über den Laser gelöst werden konnte. Mit dem Einsatz des Lasers kann völlig lotfrei gearbeitet werden, was nicht nur zu einer erheblichen Qualitätssteigerung, sondern auch zu wesentlich korrosionsresistenteren Verbindungen beiträgt. Heutige moderne Laser wie der desktop compact haben nicht nur eine große Leistungsbandbreite - sie sind

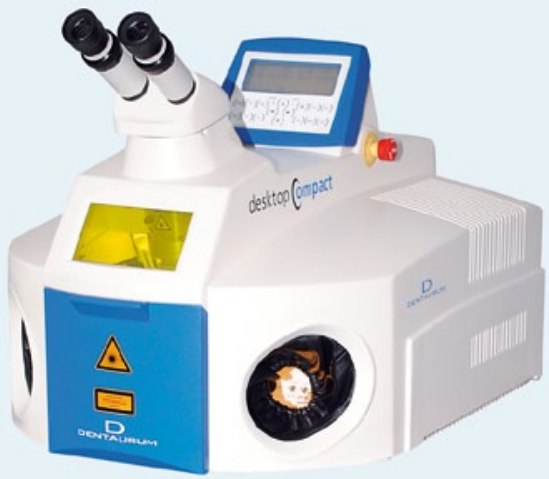

kompakt und sehr anwenderfreundlich. Feinste Schweißungen, wie z.B. bei sehr dünnen Drähten, bis hin zu massiven Verbindungen wie bei Suprakonstruktionen oder Reparaturen aller Art, sind schnell und sicher zu lösen. Die inzwischen 8 . Generation von High-Tech-Laserschweißgeräten für die Zahntechnik beinhaltet bei dem Unternehmen nicht nur eine optimale Geräteausstattung, sondern auch den kompletten zahntechnischen Service sowie den Wartungsservice des führenden Anbieters dentaler Laserfügetechnik. Weitere Informationen zu dem Dentaurum desktop Laser sind erhältlich unter: http://www.dentaurum.de/deu/schweiss geraet-16840.aspx.

Nach einer Pressemitteilung der DENTAURUM GmbH \& Co. KG, Ispringen Internet: www.dentaurum.de

\section{Praktische Komplettlo̊sung}

Wer jetzt das beliebte DC1 für die schnelle und effiziente manuelle Instrumentenaufbereitung bestellt, erhält eine praktische Filz-Handytasche gratis dazu. Damit sind nicht nur das Mobiltelefon, sondern auch die Instrumente auf der sicheren Seite, denn Komet bietet mit DC1 eine praktische Komplettlösung an: ein kombiniertes Reinigungs- und Desinfektionsmittel, das die zahnärztlichen Instrumente maximal schont, selbst wenn die Einlegezeit einmal überschritten wird. Die viruzide (wirksam entsprechend den Anforderungen des RKI gegen behüllte Viren inkl. HBV, HCV und HIV), bakterizide (inkl. TBC und MRSA) und fungizide Wirkung ist bei einer $1 \%$ igen Konzentration von DC1 nach 60 min ge- geben. Nur 5 min werden für das gleiche Ergebnis benötigt, wenn das Instrumentarium in einer 2 \%igen Lösung im Ultraschallbad gereinigt und desinfiziert wird. Das VAH/DGHM-gelistete Konzentrat ist aldehydfrei und kann somit auch für die nicht fixierende Vorreinigung eingesetzt werden. Wer sich also schlau bevorraten und sich dabei gleichzeitig eine coole Handytasche sichern will, der nimmt schnell Kontakt mit dem Komet-Fachberater auf oder ruft unter der Komet-Hotline Verkauf (05261/701-700) an.

Nach einer Pressemitteilung der

Komet Dental Gebr. Brasseler GmbH \& Co. KG, Lemgo

Internet: www.kometdental.de

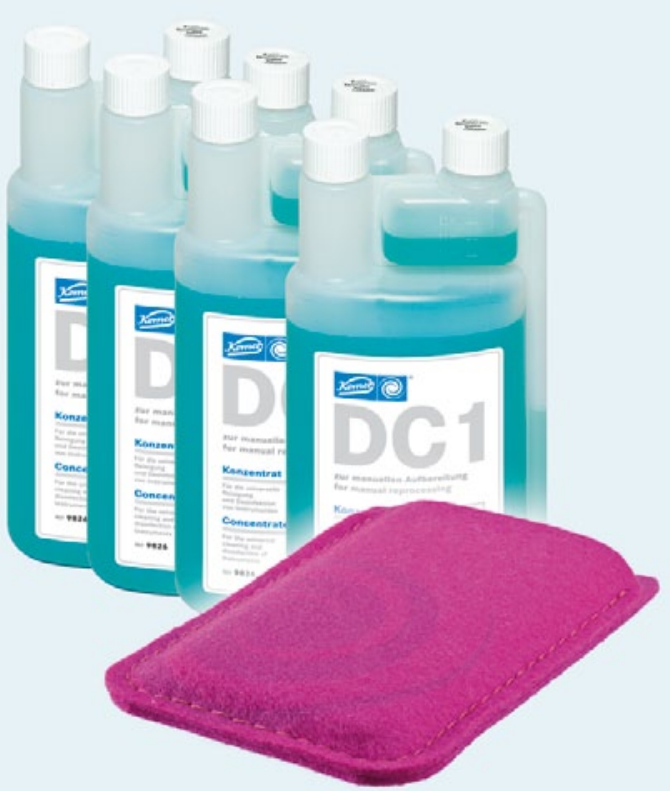




\section{Auszeichnung erhalten}

Das renommierte US-Testinstitut The Dental Advisor hat Tetric EvoCeram Bulk Fill, das Seitenzahn-Komposit von Ivoclar Vivadent, mit 5 von 5 Wertungspunkten und dem Prädikat „Editors“ Choice“ ausgezeichnet. Das Material wurde von 31 Fachpersonen für insgesamt 746 Restaurationen verwendet. Das Komposit erreichte eine klinische Bewertung von $97 \%$ mit den folgenden Resultaten: Das Material zeigt sehr gute Eigenschaften für die Anwendung im Seitenzahnbereich. Aufgrund der Durchhärtungstiefe von $4 \mathrm{~mm}$ können die meisten Kavitäten mit einer einzelnen Schicht gefüllt werden, was die Behandlungszeit verkürzt. Das Material lässt sich leicht an die Kavitätenwände adaptieren und ist gut modellierbar. Die 3 Farben sind ausreichend für die Anwendung im Seitenzahnbereich, und ihre Transluzenz sorgt für eine natürliche Anpassung an den Schmelz. In stark verfärbtem Dentin kann die Farbe durch das Komposit scheinen, wenn nicht zuvor ein opaker Liner aufgetragen wurde. Die Röntgensichtbarkeit wurde als sehr gut bewertet.

Tetric EvoCeram Bulk Fill ist ein modellierbares Nanohybrid-Komposit für die effiziente 4-mm-Bulk-Fill-Technik im Seitenzahnbereich. Das röntgensichtbare, lichthärtende Komposit enthält zusätzlich zu den konventionellen Lichtinitiatoren Campherchinon und Lucirin den patentierten Lichtinitiator Ivocerin. Im Gegensatz zu konventionellen Initiatoren ist der Polymerisations-Booster Ivocerin viel reaktiver. Das bedeutet, dass auch in tiefen Kavitäten Ivocerin aktiviert wird und das Material sicher in $10 \mathrm{~s}$ aushärtet $\left(>1000 \mathrm{~mW} / \mathrm{cm}^{2}\right)$. Dank des patentierten Licht-Controllers kann ganz ohne Zeitdruck modelliert werden. Damit auch der Schrumpfungsstress während der Polymerisation möglichst gering ausfällt, enthält das Material einen speziellen Schrumpfungsstress-Relaxator. Das Produkt ist in 3 Farben erhältlich: IVA für leicht rötliche Zähne, IVB für leicht gelbliche Zähne und IVW für Milchzähne bzw. helle Zähne.

Quelle: The Dental Advisor, Vol. 29, Nr. 05, Juni 2012

Nach einer Pressemitteilung der

Ivoclar Vivadent GmbH, Ellwangen, Jagst

Internet: www.ivoclarvivadent.de

\section{Fortbildung \\ Ein guter Weg, sich auf die Zukunft vorzubereiten}

Das umfangreiche Fortbildungsangebot der Deutschen ITI-Sektion (International Team for Implantology) geht nach den Sommermonaten in die 2. Runde. 17 weitere Seminare und Kurse werden im 2. Halbjahr 2012 angeboten.

Für den Schwerpunkt orale Chirurgie stehen den Interessenten 12 und für das Fachgebiet Prothetik/Zahntechnik 5 weitere Kurse zur Auswahl. Praktische Übungen am Tierpräparat ermöglicht das in Köln und Wolfsburg stattfindende Seminar „Parodontale Regeneration und ästhetische Rehabilitation“. Live-Operationen, Videodemonstrationen und unterstützende Fallplanung flankieren den Lernerfolg bei 3 Kursthemen, so auch im Kurs „Wie unterstützen computerassistierte Planung und Guided Surgery den Therapieerfolg in der Implantologie?“. Die ganztägigen Seminare mit dem Fokus auf Prothetik und Zahntechnik bieten laut ITI die Gelegenheit, Versorgungskonzepte und Planungsgrundla- gen kritisch zu betrachten, zu bewerten, zu erlernen und zu perfektionieren.

Die SAC-Klassifizierung ermöglicht einen schnellen Überblick und erleichtere die Entscheidung für die Buchung. Nach „Standard“, „Anspruchsvoll“ und „Komplex“ gekennzeichnet, bietet die Zusammenstellung des Fortbildungsangebots Kurse für Anwender jeder Erfahrungsund Behandlungskategorie.

Das ITI empfiehlt, sich die 40-seitige Fortbildungsbroschüre $\mathrm{zu}$ bestellen und vor jedem Kurs das Detailprogramm anzufordern, da die Referenten unterschiedliche Schwerpunkte legen und die Inhalte laufend aktualisiert werden.

Weitere Informationen und Anmeldung bei ITI Sektion Deutschland

Jechtinger Straße 9, 79111 Freiburg

E-Mail: germany@itisection.org

\section{Küretten \\ Neuheit bei Küretten}

Der Hersteller American Eagle stellt eine Innovation vor: die neuen Double-Gracey-Küretten, eine Kombination aus mehreren Gracey-Varianten. Sie haben eine Doppelklinge und sind mit ihren 2 Schneiden als Universalinstrument für alle Wurzelbereiche einsetzbar.

Mit nur 2 Instrumenten können nun sämtliche Wurzeloberflächen effektiv kürettiert werden. Die beiden Double-Gracey-Instrumente Anterior und Posterior werden anstelle der klassischen Gracey- und Universalküretten verwendet. Zwei weitere „Mini“-Instrumente mit einer um 50\% kürzeren Klinge und einem $3 \mathrm{~mm}$ längeren Schaft sorgen für besseren Zugang in tiefen Taschen und engen Wurzelbereichen.

Die aufwendige Prozedur der Kürettage wird für den Behandler wesentlich einfacher. Häufiger Instrumentenwechsel, Kodierung verschiedener Spitzenvarianten und die kostenintensive Lagerhaltung mehrerer Instrumentensätze gehören der Vergangenheit an. Der Einsatz der DoubleGracey-Küretten ermöglicht ein effektives,

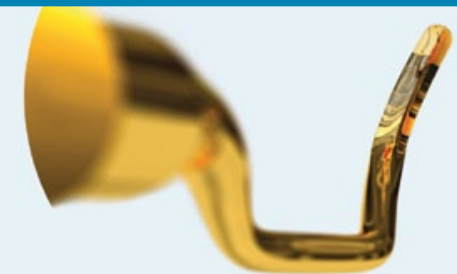

zeitsparendes Debridement und macht den Einsatz der Instrumente auch aus wirtschaftlichen Aspekten interessant.

Die Entwicklung der Instrumente wurde erst durch die XP-Technologie möglich. Wie alle anderen XP-Instrumente von American Eagle müssen Double Graceys während ihrer Einsatzzeit nicht geschliffen werden. Die speziell gestalteten Arbeitsenden der Küretten würden durch regelmäßiges Schleifen ihre ursprüngliche Form und Effektivität schnell verlieren. Erst mit der nanooptimierten XP-Oberfläche kann das aufwendig gestaltete Schneidekantendesign der Doppelklingen dauerhaft erhalten werden.

Die Instrumente sind ab sofort über den Handel zu beziehen.

Nach einer Pressemitteilung der
Loser \& Co, Leverkusen

Internet: www.loser.de 


\section{Exaktes Design und komfortable Fertigung aus einer Hand}

Für ein flexibleres computergestütztes Gestalten und Fräsen von Kronen und Brücken, hat Straumann sein neues CARES CAD/CAM-System weiter optimiert. So unterstützt die Software 2 unterschiedliche Produktionsphilosophien. Laut dem Unternehmen bietet das CARES-System 7.0 durch seinen validated Workflow (interner Arbeitsablauf) Prothetikelemente von gleichbleibend hoher Qualität und Präzision. Gleichzeitig ermöglicht es seinen Nutzern, flexibel auf external Workflows (externer Arbeitsablauf) zuzugreifen.

Beim validierten Arbeitsablauf mit direkter Anbindung zum Straumann-Fräszentrum sind die Parameter bereits voreingestellt. Somit erhalte der Anwender geprüfte, zuverlässige und vorhersagbare Produktergebnisse mit exaktem Design und sehr guter Passform.
Die neue Software kombiniert ein breites Anwendungs- und Materialspektrum. So lassen sich Patienten beispielsweise mit Inlays, vollanatomischen Kronen und individueller Implantatprothetik versorgen. Auch 16-gliedrige Brückengerüste sind möglich. Je nach Indikation können vollkeramische Materialien wie Zirkoniumdioxid, Leuchit-Glaskeramik und Feldspat-, Lithiumdisilikat- oder auch Resin NanoKeramik verarbeitet werden. Ebenso stehen metallische Werkstoffe wie Kobalt-ChromLegierungen oder Titan sowie verschiedene Kunststoffe zur Verfügung.

Nach einer Pressemitteilung der

Straumann GmbH, Freiburg

Internet: www.straumann.de

\section{Fünf aktuelle Awards}

Der bewährte GLUMA ${ }^{\circledR}$ Desensitizer von Heraeus wird dieses Jahr erneut in seiner Rolle als Vorreiter bei der Behandlung postoperativer Sensibilitäten bestätigt. Auch das neue GLUMA $^{\circledR}$ Desensitizer PowerGel wird 2012, wie schon 2011, von renommierten amerikanischen Fachmedien prämiert. Vom unabhängigen Testinstitut „The Dental Advisor“ erhält es neben dem „Preferred Products 2012“-Award die Auszeichnung „2012 Clinical Problem Solver“. Darüber hinaus erhielt der neue Desensibilisierer schon 2011 ein 5-Diamond-Rating als „Contemporary Product Solution“ des gleichnamigen Fachmagazins. Als die mit Abstand bekannteste Marke unter den Desensibilisierern wird GLUMA ${ }^{\circledR}$ auch 2012 mit dem Online - „Townie Choice Award“ ausgezeichnet. GLUMA ${ }^{\circledR}$ Desensitizer Liquid erhält zudem auch 2012 den „Reality Award“ und wurde im Clinicians Report unter den „best products of 2011“ gelistet. GLUMA $^{\circledR}$ Desensitizer PowerGel zur Vermeidung von Dentinhypersensibilitäten ist seit Beginn des Jahres auch in Europa erhältlich. Es überzeugt wie GLUMA ${ }^{\circledast}$ Desensitizer durch effektive Wirksamkeit und sofortige Schmerzreduktion. Die gelartige Konsistenz und seine grüne Farbe ermöglichen eine punktgenaue und kontrollierte Applikation und damit vereinfachtes Auftragen. Das schafft eine höhere

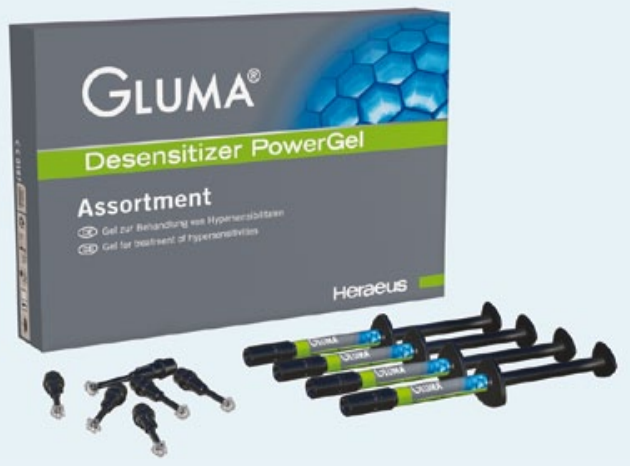

Präzision und minimiert das potenzielle Risiko des Kontakts mit dem Weichgewebe. Durch die hohe Effektivität des PowerGels ist keine wiederholte Anwendung erforderlich. Dies und die Ein-Schritt-Applikation ohne Anmischen oder Lichtpolymerisation führen zu einer Zeit- und Kostenersparnis. Das neue PowerGel ist zudem mit allen marktüblichen Bonding-Systemen kompatibel.

Das Gel wird zur sofortigen und langfristigen Schmerzausschaltung bzw. Schmerzreduktion bei freiliegenden Zahnhalsbereichen verwendet. Eine weitere Indikation ist die Anwendung an präparierten Zähnen vor dem Einbringen direkter oder indirekter Restaurationen. Hier wird es zur Vermeidung postoperativer Sensibilitäten und Schmerzen eingesetzt.

Nach einer Pressemitteilung der Heraeus Kulzer GmbH, Hanau

Internet: www.heraeus-dental.com 